hep-th/0103264

\title{
Path integral formulation of Hodge duality on the brane
}

\author{
Sang-Ok Hahn周, Youngjai Kiem[, Yoonbai Kimf, and Phillial Oh円 \\ BK21 Physics Research Division and Institute of Basic Science, Sungkyunkwan University, \\ Suwon 440-746, Korea
}

\begin{abstract}
In the warped compactification with a single Randall-Sundrum brane, a puzzling claim has been made that scalar fields can be bound to the brane but their Hodge dual higher-rank antisymmetric tensors cannot. By explicitly requiring the Hodge duality, a prescription to resolve this puzzle was recently proposed by Duff and Liu. In this note, we implement the Hodge duality via path integral formulation in the presence of the background gravity fields of warped compactifications. It is shown that the prescription of Duff and Liu can be naturally understood within this framework.
\end{abstract}

\footnotetext{
*hahnenewton.skku.ac.kr

†ykiemenewton.skku.ac.kr

$\ddagger$ yoonbai@skku.ac.kr

${ }^{\S}$ ploh@newton.skku.ac.kr
} 
The warped space-time geometry of Randall-Sundrum brane world in $(d+1)$-dimensions, whose world-volume is $d$-dimensional, is described by the following metric:

$$
d s^{2}=\hat{g}_{M N} d X^{M} d X^{N}=\exp (-2 k|z|) g_{\mu \nu}\left(x^{\mu}\right) d x^{\mu} d x^{\nu}+d z^{2} .
$$

The coordinates of the bulk space-time will be denoted as $X^{M}$, the coordinates parallel to the brane, $x^{\mu}$, and the direction perpendicular to the brane will be parametrized by the coordinate $z$. The brane is assumed to be located at $z=0$. In the brane world scenario with two branes [1], a natural geometric framework to deal with the gauge hierarchy problem emerges as a consequence of the the exponential warp factor in (11). After a wave-function renormalization, it produces an extremely tiny number $m_{\mathrm{EW}} / M_{\text {Planck }} \sim 10^{-16}$ when the distance between the branes is about 40 times the Planck scale. The warp factor in the Jacobian $\sqrt{-\operatorname{det} \hat{g}_{M N}}$ allows normalizable gravitational zero modes localized on a single brane when the range of $z$ is extended to $z=\infty$ [2].

All these attractive features are due to the exponential suppression in the large $z$ region coming from the covariant metric components $\hat{g}_{M N}$. An opposite situation, however, might result when the contravariant metric components $\hat{g}^{M N}$ are involved in the classical action. A particularly intriguing case involves the anti-symmetric tensor fields of various ranks and their Hodge duals. Since the classical action integral over the $z$ direction is finite, a scalar field can be bound to the brane. Higher rank anti-symmetric tensor fields that are Hodge dual to scalars cannot however be bound to the brane, since the similar integral diverges due to the large number of contravariant metric components needed to contract the field strengths [3, \#]. To resolve this apparent violation of the Hodge duality, a proposal for a proper Kaluza-Klein prescription was suggested by Duff and Liu [5]. They found an appropriate Kaluza-Klein ansatz for higher rank anti-symmetric tensor fields, which explicitly satisfies the Hodge duality at the classical level. In the case of conventional (curved) space-time examples, a fool-proof and straightforward approach to implement the Hodge duality is to formulate it in terms of path integrals [6]. In the same spirit, we implement the Hodge duality in the warped background geometry described by (11) via the path integral formalism. We find that the prescription of [5] can be naturally obtained in this fashion.

The standard diffeomorphism invariant action for the $p$-form anti-symmetric tensor gauge 
field $\hat{A}\left(X^{M}\right)=\hat{A}_{[p]}\left(x^{\mu}, z\right)$ is

$$
S_{F}=-\frac{1}{2(p+1) !} \int d^{d+1} X \sqrt{-\hat{g}}\left(\hat{g}^{M_{1} N_{1}} \cdots \hat{g}^{M_{p+1} N_{p+1}} \hat{F}_{M_{1} \cdots M_{p+1}} \hat{F}_{N_{1} \cdots N_{p+1}}\right)
$$

To find a sensible Kaluza-Klein ansatz, we consider the solutions to the parts of equations of motion

$$
\partial_{z}\left(\sqrt{-\hat{g}} \hat{g}^{z z} \hat{g}^{\mu_{1} \nu_{1}} \cdots \hat{g}^{\mu_{p} \nu_{p}} \hat{F}_{z\left[\nu_{1} \cdots \nu_{p}\right]}\right)=0,
$$

which should be satisfied for the Kaluza-Klein zero modes. Two consistent solutions to (3) have been discussed in Refs. [3, 4, 5]. To be specific, an ansatz is a zero-mode solution

$$
\hat{A}_{\mu_{1} \cdots \mu_{p}}\left(x^{\mu}, z\right)=A_{\mu_{1} \cdots \mu_{p}}\left(x^{\mu}\right)
$$

and plugging it into (2) along with the background metric produces the action

$$
\begin{aligned}
S_{F}= & -\frac{1}{2(p+1) !} \int d z \exp \left[-2 k\left(\frac{d}{2}-p-1\right)|z|\right] \\
& \times \int d^{d} x \sqrt{-g}\left(g^{\mu_{1} \nu_{1}} \cdots g^{\mu_{p+1} \nu_{p+1}} F_{\mu_{1} \cdots \mu_{p+1}} F_{\nu_{1} \cdots \nu_{p+1}}\right) .
\end{aligned}
$$

The other ansatz is another consistent zero mode solution

$$
\hat{A}_{z \mu_{1} \cdots \mu_{p-1}}\left(x^{\mu}, z\right)=\exp \left[-2 k\left(p-\frac{d}{2}\right) k|z|\right] A_{\mu_{1} \cdots \mu_{p-1}}\left(x^{\mu}\right),
$$

which leads to

$$
\begin{aligned}
S_{F}= & -\frac{1}{2(p+1) !} \int d z \exp \left[-2 k\left(p-\frac{d}{2}\right)|z|\right] \\
& \times \int d^{d} x \sqrt{-g}\left(g^{\mu_{1} \nu_{1}} \cdots g^{\mu_{p} \nu_{p}} F_{z \mu_{1} \cdots \mu_{p}} F_{z \nu_{1} \cdots \nu_{p}}\right)
\end{aligned}
$$

upon following the same procedure. The factors containing the $z$-integral in (5) and (7) are effectively the inverse coupling squared, $1 / e_{\text {eff }}^{2}$, in the $d$-dimensional brane theory. The integral in (5) is convergent only for low values of $p$ 's that satisfy

$$
p<\frac{d-2}{2}
$$

Therefore the bulk action (5) is sensible as the brane action only for the $p$-form gauge fields satisfying the condition (8). Otherwise the effective coupling $e_{\text {eff }}$ vanishes and the gauge dynamics

\footnotetext{
${ }^{1}$ The material in this paragraph is based on our understanding of the discussions with Hyung Do Kim.
} 
on the brane appears to be decoupled. Under the condition (8), the $z$-integral in the action (7) diverges, making it problematic. For the higher $p$-form gauge fields with

$$
p>d / 2
$$

the situation for each ansatz reverses itself. While the $z$-integral in the action (5) diverges, the $z$-integral in the other action (7) converges. For the marginal cases which do not satisfy (8) and (9), such as $p=(d-2) / 2$ and $p=d / 2$ for even $d$, the divergence coming from the $z$ integral is linear in the cutoff $z_{0}$, which translates to the logarithmic divergence $\log p_{0}$ in terms of the momentum cutoff $p_{0}$ of the $d$-dimensional brane theory. In [4], this was interpreted as the quantum charge screening effect in the brane theory (see however [5]). In this paper, we will not consider these marginal cases.

This aspect appears rather puzzling if we use the ansatz (đ) for all values of $p$; the Hodge duality in the bulk space-time relates the bulk $p$-form gauge field with a $p^{\prime}$-form gauge field satisfying

$$
p^{\prime}=d-p-1
$$

When viewed from its Hodge dual side, the $p>d / 2$ case should present no problem. Given this situation, the prescription of Duff and Liu [5] is that one should use the ansatz (6) for the $p$-form fields for $p>d / 2$. In what follows, we derive the action for the $p$-form gauge field with $p>d / 2$ from the action (5) of the $p$-form gauge field with $p<(d-2) / 2$ by implementing the Hodge duality in the path integral formalism [6]. We find that this analysis precisely reproduces the prescription given by Duff and Liu [5], which states that the ansatz (4) is relevant for $p<(d-2) / 2$ and the ansatz (6) should be used for for $p>d / 2$.

The partition function for the brane world system includes the following factor among others:

$$
\int\left[\prod_{X>0} \mathcal{D} \hat{A}_{[p]}(X)\right] \exp \left(i S_{F}\right)=\int\left[\prod_{X>0} \mathcal{D} \hat{A}_{[p]}(X)\right] \exp \left(-\frac{i}{2} \int d^{d+1} X \hat{F}_{I} A_{I J} \hat{F}_{J}\right),
$$

where

$$
\frac{1}{2(p+1) !} \sqrt{-\hat{g}} \hat{g}^{\mu_{1} \nu_{1}} \cdots \hat{g}^{\mu_{p+1} \nu_{p+1}} \hat{F}_{\mu_{1} \cdots \mu_{p+1}} \hat{F}_{\nu_{1} \cdots \nu_{p+1}}=\frac{1}{2} \hat{F}_{I} A_{I J} \hat{F}_{J}
$$

Each index $I$ collectively denotes the $(p+1)$-distinctive directions chosen from the $d$ space-time directions parallel to the brane: $I=\left\{\mu_{1}^{I}, \cdots, \mu_{p+1}^{I}\right\}$. The matrix $A_{I J}$ can thus be written as

$$
A_{I J}=\sqrt{-\hat{g}} \hat{g}^{\mu_{1}^{I} \mu_{1}^{J}} \cdots \hat{g}^{\mu_{p+1}^{I} \mu_{p+1}^{J}}
$$


The set $\left\{F_{I} \mid\right.$ all $\left.I\right\}$ has $D \equiv{ }_{d} C_{p+1}$ elements. The underlying bulk space-time geometry in the brane world setup is invariant under the $\mathbb{Z}_{2}$ transformation, whose action on $z$ is $z \rightarrow-z$, and the fixed points at $z=0$ correspond to the 'world brane'. Accordingly, the path integral in (11) is over the space-time points $X$ whose $z$-value is larger than zero with an understanding that the fluctuations of fields at $X(-z)$ are the 'mirror images' of the fluctuations at the point $X(z)$. For the gauge fields which do not have the nonzero component along the $z$-direction, the zero mode part of $\partial_{z}^{2}$ is a constant over the whole range of the space $\mathbb{R}^{1, d-1} \times \mathbb{R}$, as shown in the standard Kaluza-Klein ansatz $\hat{A}_{\mu_{1} \cdots \mu_{p}}\left(x^{\mu}, z\right)=A_{\mu_{1} \cdots \mu_{p}}\left(x^{\mu}\right)$.

The Hodge duality is implemented at the level of the path integral by replacing the exponential function appearing in (11) via an identity

$$
\begin{gathered}
\exp \left[-\frac{i}{2} \int d^{d+1} X \hat{F}_{I} A_{I J} \hat{F}_{J}\right]=\int\left[\prod_{X>0}\left[\operatorname{det} A_{I J}(X)\right]^{-1} \prod_{I} \mathcal{D} \hat{\tilde{F}}_{I}(X)\right] \\
\quad \times \exp \left[\frac{i}{2} \int d^{d+1} X \hat{\tilde{F}}_{I}\left(A^{-1}\right)_{I J} \hat{\tilde{F}}_{J}+i \int d^{d+1} X \hat{\tilde{F}}_{I} \hat{F}_{I}\right]
\end{gathered}
$$

up to an overall numerical factor that we neglect from here on. The symbol $\prod_{X>0}$ again signifies the fact that the functional integral is over the bulk space-time points $X$ on $\mathbf{R}^{1, d-1} \times \mathbb{R}^{+}$, namely, the points $X$ whose $z$ coordinates satisfy $z>0$. The rationale behind this choice becomes clearer when we consider the zero modes of the Hodge dual fields. When the KaluzaKlein ansatz for the original gauge field is of the form in (†), its non-vanishing Hodge dual field strength $\hat{\tilde{F}}$ necessarily involves the $z$-direction, or in other words, the components $\hat{\tilde{F}}_{\mu_{1} \cdots \mu_{p^{\prime}} z} \neq 0$, where $p^{\prime}=d-p-1$. Assuming their intrinsic parity under $\mathbb{Z}_{2}$ is even?, the components $\hat{\tilde{F}}_{\mu_{1} \cdots \mu_{p^{\prime}} z}$ transform to $-\hat{\tilde{F}}_{\mu_{1} \cdots \mu_{p^{\prime}}}$ under the $\mathbb{Z}_{2}$ transformation $z \rightarrow-z$. The components $\hat{\tilde{F}}_{\mu_{1} \cdots \mu_{p^{\prime}} z}$ thus contain the factor $\operatorname{sgn}(z)$; due to the discontinuity at $z=0$, the components $\hat{\tilde{F}}_{\mu_{1} \cdots \mu_{p^{\prime}} z}$ are regular only in $\mathbf{R}^{1, d-1} \times \mathbf{R}^{+}$. Under our prescription, however, the path integral is only over the space-time region where the dual gauge fields are well-defined (regular), leading to (14). The consequence of this prescription is that the power of $\operatorname{det} A_{I J}(X)$ in (14) is -1 , unlike the case when the domain of the functional integration is over the whole simple covering space $\mathbf{R}^{1, d-1} \times \mathbf{R}$, which would lead to $-1 / 2$ in (14).

The number of independent components of $\hat{\tilde{F}}_{I}$ is ${ }_{d} C_{p^{\prime}}={ }_{d} C_{d-p-1}={ }_{d} C_{p+1}=D$, which is the same number as the independent components of $\hat{F}_{I}$. By a direct combinatorics consider-

\footnotetext{
${ }^{2}$ There exist bulk higher form gauge fields with negative intrinsic parity in string/M theory. For these fields, more refined arguments are needed.
} 
ation, one can show that

$$
\frac{1}{2} \hat{\tilde{F}}_{I}\left(A^{-1}\right)_{I J} \hat{\tilde{F}}_{J}=\frac{1}{2\left(p^{\prime}+1\right) !} \sqrt{-\hat{g}} \hat{g}^{\mu_{1} \nu_{1}} \cdots \hat{g}^{\mu_{p^{\prime}} \nu_{p^{\prime}}} \hat{g}^{z z} \hat{\tilde{F}}_{\mu_{1} \cdots \mu_{p^{\prime}}} \hat{\tilde{F}}_{\nu_{1} \cdots \nu_{p^{\prime}} z}
$$

and

$$
\operatorname{det} A_{I J}=(-\hat{g})^{\frac{D}{2}-\frac{D}{d}(p+1)}=(-\hat{g})^{\frac{D}{2 d}(d-2 p-2)}=(-\hat{g})^{\frac{D}{d}\left(p^{\prime}-d / 2\right)}
$$

using $\hat{g}_{z z}=1$ and $\hat{g}_{\mu z}=0$. We observe that (15) is the standard kinetic term for the $p^{\prime}$-form gauge field. We use the classical approximation for the path integral over the metric by inserting the background metric (11) into the path integral (14). For the metric of the type det $g=1$, the measure part of (14) can be rewritten as

$$
\left[\prod_{X>0}\left[\operatorname{det} A_{I J}(x)\right]^{-1} \prod_{I} \mathcal{D} \hat{\tilde{F}}_{I}(X)\right]=\left[\prod_{X>0} \prod_{I} \exp \left[2 k\left(p^{\prime}-d / 2\right)|z|\right] \mathcal{D} \hat{\tilde{F}}_{I}(X)\right] .
$$

The path integral over the $\mathcal{D} \hat{A}_{[p]}$ in (11), due to the $\hat{\tilde{F}}_{I} \hat{F}_{I}$ term in (14), imposes the usual constraint $d \hat{\tilde{F}}=0$ for the functional integral, which implies that only the field strength that can be written as $\hat{\tilde{F}}=d \hat{\tilde{A}}_{\left[p^{\prime}\right]}$ contributes to the path integral. The only difference between the conventional Hodge dual action in the flat space and the case in consideration is the extra $z$-dependence in the path integral measure (17). By writing, however,

$$
\hat{\tilde{A}}_{\mu_{1} \cdots \mu_{p^{\prime}-1} z}=\exp \left[-2 k\left(p^{\prime}-d / 2\right)|z|\right] \tilde{A}_{\mu_{1} \cdots \mu_{p^{\prime}-1} z}
$$

we can transform the measure part (17) into

$$
\left[\prod_{X>0}\left[\operatorname{det} A_{I J}(X)\right]^{-1} \prod_{I} \mathcal{D} \hat{\tilde{F}}_{I}(X)\right]=\left[\prod_{X>0} \prod_{I} \mathcal{D} \tilde{F}_{I}(X)\right] .
$$

We note that $d \hat{\tilde{A}}_{\left[p^{\prime}\right]}=0$ automatically implies $d \tilde{A}_{\left[p^{\prime}\right]}=0$. Our main result is that (18) is precisely the Kaluza-Klein ansatz appearing in (7) of Ref. [5] for the $p$-form gauge fields with $p>d / 2$.

In this note, we have shown that the higher form gauge fields that are Hodge dual to the gauge fields that can survive on the brane also survive on the brane. Restricting our attention only to 'fundamental' gauge fields that have the conventional Kaluza-Klein ansatz, however, the condition (8) still applies. It is amusing to note that the 'fundamental' higher form gauge fields do not live on the brane world, which happens to be the property of our world.

Our analysis can be generalized in various directions. One can, for example, repeat the same type of analysis in the context of higher (transversal) dimensional warped compactifications, where the background metric is given by

$$
d s^{2}=\hat{g}_{M N} d X^{M} d X^{N}=\exp (-2 k r) g_{\mu \nu}\left(x^{\mu}\right) d x^{\mu} d x^{\nu}+d r^{2}+C^{2}(r) d \Omega_{N-1} \text {. }
$$


These background geometries are typically classified into two categories; the cigar geometries of constant radius, $C(r)=C_{\infty}$, and bottle-neck geometries of exponentially decreasing radius, $C(r)=e^{-k r}$ [7]. These two cases produce different classical action and different Jacobian factor in the path integral measure after taking Hodge duality. Comparing them and finding correct Kaluza-Klein ansatz should be an interesting issue [8]. Secondly, in view of the stringinspired model constructions, it is important to consider the bulk gauge fields with odd intrinsic parity.

\section{Acknowledgements}

The authors would like to thank Jin-Ho Cho and Hyung Do Kim for useful discussion. We are also grateful to M.J. Duff and J.T. Liu for encouraging correspondence. This work is supported by No. 2000-1-11200-001-3 from the Basic Research Program of the Korea Science \& Engineering Foundation and Korea Research Center for Theoretical Physics and Chemistry (Y. Kim).

\section{References}

[1] L. Randall and R. Sundrum, Phys. Rev. Lett. 83, 3370 (1999), hep-th/9905221.

[2] L. Randall and R. Sundrum, Phys. Rev. Lett. 83, 4690 (1999), hep-th/9906064.

[3] N. Kaloper, E. Silverstein, and L. Susskind, hep-th/0006192.

[4] E. Silverstein, Proceedings of Strings 2000, Int. J. Mod. Phys. 16, 641 (2001), hepth/0010144.

[5] M.J. Duff and J.T. Liu, hep-th/0010171.

[6] C. Kim and Y. Kim, Phys. Rev. D50, 1040 (1994), hep-th/9401113; N. Kim, Y. Kim, and K. Kimm, Phys. Rev. D56, 8029 (1997), gr-qc/9707056.

[7] R. Gregory, Phys. Rev. Lett. 84, 2564 (2000), hep-th/9911015.

[8] S.-O. Hahn, C. O. Lee, and H.-S. Yoon, in preparation. 